\title{
Discrete self--organizing migration algorithm and p-location problems
}

\author{
Jaroslav Janáček ${ }^{1, *}$ and Marek Kvet ${ }^{1}$ \\ ${ }^{1}$ Faculty of Management Science and Informatics, University of Žilina \\ Univerzitná 8215/1, 01026 Žilina, Slovakia \\ E-mail: 〈\{jaroslav.janacek, marek.kvet\}@fri.uniza.sk〉
}

\begin{abstract}
Mathematical modelling, and integer programming generally, has many practical applications in different areas of human life. Effective and fast solving approaches for various optimization problems play an important role in the decision-making process and therefore, big attention is paid to the development of many exact and approximate algorithms. This paper deals only with a special class of location problems in which given number of facilities are to be chosen to minimize the objective function value. Since the exact methods are not suitable for their unpredictable computational time or memory demands, we focus here on possible usage of a special type of a particle swarm optimization algorithm transformed by discretization and meme usage into so-called discrete self-organizing migrating algorithm. In the paper, there is confirmed that it is possible to suggest a sophisticated heuristic for zero-one programming problem, which can produce near-to-optimal solution in much smaller time than the time demanded by exact methods. We introduce a special adaptation of the discrete selforganizing migration algorithm to the $p$-location problem making use of the path-relinking method. In the theoretical part of this paper, we introduce several strategies of the migration process. To verify their features and effectiveness, a computational study with real-sized benchmarks was performed. The main goal of the experiments was to find the most efficient version of the suggested solving tool.
\end{abstract}

Keywords: discrete self-organizing migration algorithm, large location problems, meme usage, pathrelinking method

Received: September 02, 2020; accepted: November 19, 2020; available online: December 18, 2020

DOI: $10.17535 /$ crorr.2020.0019

\section{Introduction}

Many kinds of public service systems are usually designed to satisfy their users by providing necessary service in case of emergency. Since the resources, from which the service can be provided, are limited mostly due to economic or technological restrictions, the mathematical model of the problem may often take the form of a weighted $p$-median problem $[3,7,18,21]$ or any similar one. To make the mathematical model applicable in wider spectrum of research problems, the concept of so-called generalized disutility has been introduced [8, 13, 15, 19, 26]. This model extension enables to cover more demands of system users, which can arise almost simultaneously, and furthermore, the nearest located service center does not need to have sufficient capacity to solve all the assigned demands at the same time. Of course, mentioned model generalization brings some difficulties into the solving process of the problem.

The necessity to solve large problem instances has led to the development of several effective solving techniques. The first group of approaches contains the exact methods based on the branch and bound principle. Many of them use a specific model reformulation, in which socalled radial approach is applied $[1,3,9,10,24]$. One of the biggest disadvantages of the

${ }^{*}$ Corresponding author. 
exact methods consists in their almost unpredictable demands, which usually make the solving process extremely time consuming. Simultaneously, many different approximate methods have been developed. Currently, the main attention is paid to various metaheuristic approaches, i.e. genetic algorithms, scatter search, path-relinking method and many others [11, 12, 14, 23], the aim of which can be specified as a task of obtaining a good solution in acceptably short time.

In this paper, we focus on study of possible usage of a special type of a particle swarm optimization algorithm [2] transformed by discretization and meme usage into so-called discrete self-organizing migrating algorithm $[5,22,30]$.

The principle of the self-organizing migrating algorithm has proved to be very hopeful tool for solving real life problems from the field of engineering [6, 25]. The discrete version of the selforganizing migrating algorithm has been successful in solving complicated problems described as zero-one programming problems in the field of scheduling and routing [4]. The successful results in solving real complex discrete problems indicate that the principle can be also used to solve other scheduling problems referenced in [27, 28, 29]. Nevertheless, it must be taken into account that a successful application of any metaheuristic must be paid by very thoroughly performed implementation of the solving algorithm making use of individual characteristics of the problem.

In this paper, we deal with the hypotheses that stochastic characteristic of service of randomly emerging demands including impact of limited capacity of service centers can be described using generalized disutility model and, furthermore, that it is possible to suggest a sophisticated heuristic for a specific problem, which can produce near-to-optimal solution in much smaller time than the time demanded by an exact method.

The study is aimed at application of the algorithm to efficient solving of the min-sum $p$ location problem. We introduce a special adaptation of the discrete self-organizing migration algorithm to the $p$-location problem making use of the path-relinking method. We study various strategies of the migration process and compare them to find the most efficient version of the solving tool.

\section{Strategies of discrete self-organizing migrating algorithm}

The standard general particle swarm optimization algorithm repeatedly updates a population (swarm) of problem solutions (particle positions) by performing a perturbation with each solution of the population taking into account the general best-found solution and also the best solutions in trajectories traversed by the individual particles. The way of perturbation depends on the range of the optimized objective function and the perturbation operations include weights of the general and local best-found solutions and some random changes. The derived discrete self-organizing migrating algorithm is also based on the idea of updating particle positions corresponding to problem solutions, nevertheless updating of an individual particle is performed by moving its current position to the best position found on the trajectory connecting the current position of the updated particle to some other particle. The choice of the other particle is specified by the used migration strategy. Based on the way of population updating, various strategies can be distinguished. We will study the following often referred ones [30].

The strategy AllToOne (ATO): Each particle of the current population is evaluated and the best one is denoted as a leader. Then the trajectories from the individual particles to the leader are inspected and the particle positions are updated with exception of the leader. Updated particle positions and the leader position represent the new population.

The strategy AllToAll (ATA): This strategy does not distinguish any special particle, e.g. the leader. Each trajectory from an updated particle to other particle locations is inspected and the best found positions enter the new population.

The above mentioned strategies have also their adaptive versions, which follow. 
The strategy AllToOne-Adaptive $(A T O-A)$ : This strategy starts similarly to ATO by population evaluation and the leader determination. Then, trajectories from particles to the leader are subsequently inspected and the position of the leader is updated immediately after each trajectory inspection.

The strategy AllToAll-Adaptive $(A T A-A)$ : This strategy differs from the above-described $A T A$ only in the start of a single trajectory inspection. When trajectories from an updated particle are inspected, the starting position corresponding to the particle is updated after each trajectory inspection. A decision on the most efficient strategy for the studied $p$-location problem is matter of experiments, which will be referred in the remainder of the paper.

\section{Implementations of the discrete self-organizing migrating algorithm for the min-sum $p$-location problem}

The solved $p$-location problem defined for $m$ possible facility locations and $p$ facilities to be located can be described by (1) in general.

$$
\min \{f(P), P \subset\{1,2, \ldots, m\},|P|=p\}
$$

The generalized min-sum version of the $p$-location problem is determined by the objective function $f$ specification (2).

$$
f(P)=\sum_{j=1}^{n} b_{j} \sum_{k=1}^{r} q_{k} \min _{k}\left\{d_{i j}: i \in P\right\}
$$

The objective function value $f(P)$ for given subset $P$ of facility locations is computed for $n$ users with weights $b_{j}$ for $j=1, \ldots, n$ and for probability values $q_{k}$ for $k=1, \ldots, r$, where $q_{k}$ is probability that the $k$-th nearest facility is the closest available one. The symbol $d_{i j}$ stays for the distance between locations $i$ and $j$ and operation $\min _{k}\{\}$ denotes the $k$-th minimal value of the list of values in the brackets.

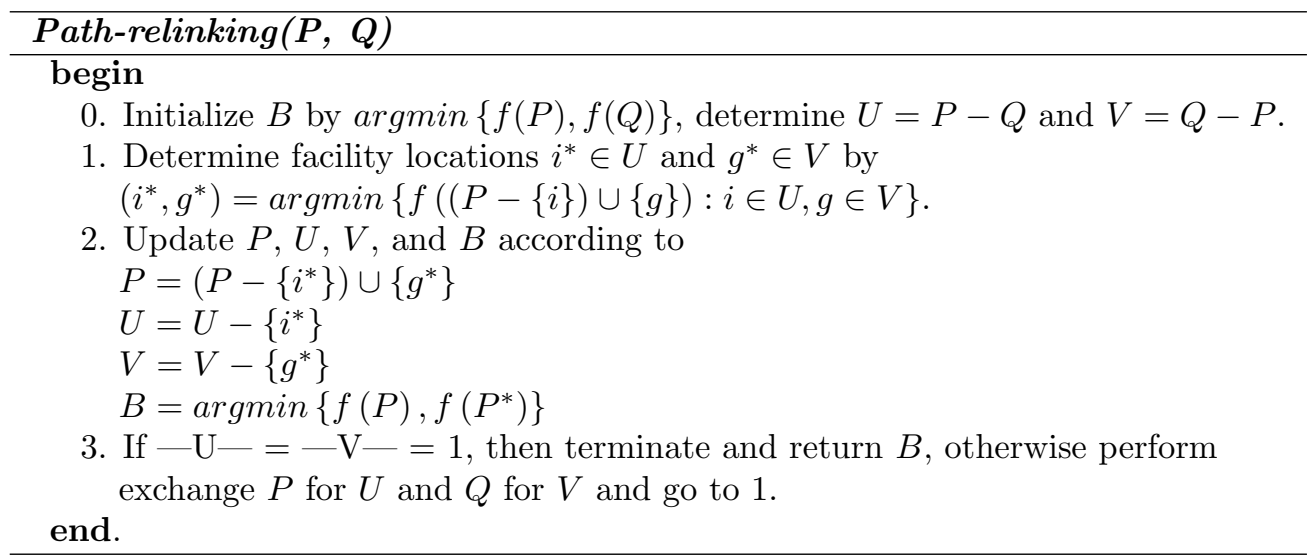

Table 1: Algorithm Path-relinking $(P, Q)$

The presented implementations of the discrete self-organizing migrating algorithms are based on the path-relinking method, implementation of which makes use of a special structure of the set of all feasible solutions of (1). The method inspects vertices of an $m$-dimensional hypercube lying on the shortest path in the hypercube surface between elements of a pair of input vertices corresponding with a pair of the $p$-location problem solutions. The path-relinking method 
$[16,20]$ skips the hypercube vertices, which do not correspond to feasible solutions of (1), i.e. the vertices, where $|P| \neq p$. Let $P$ and $Q$ denote a pair of input solutions. Then, the used path-relinking method is described by the following steps.

Let $S=\left\{P^{1}, \ldots, P^{|S|}\right\}$ denote a starting population of feasible solutions of the problem (1). Descriptions of the studied implementations associated with the individual strategies follow.

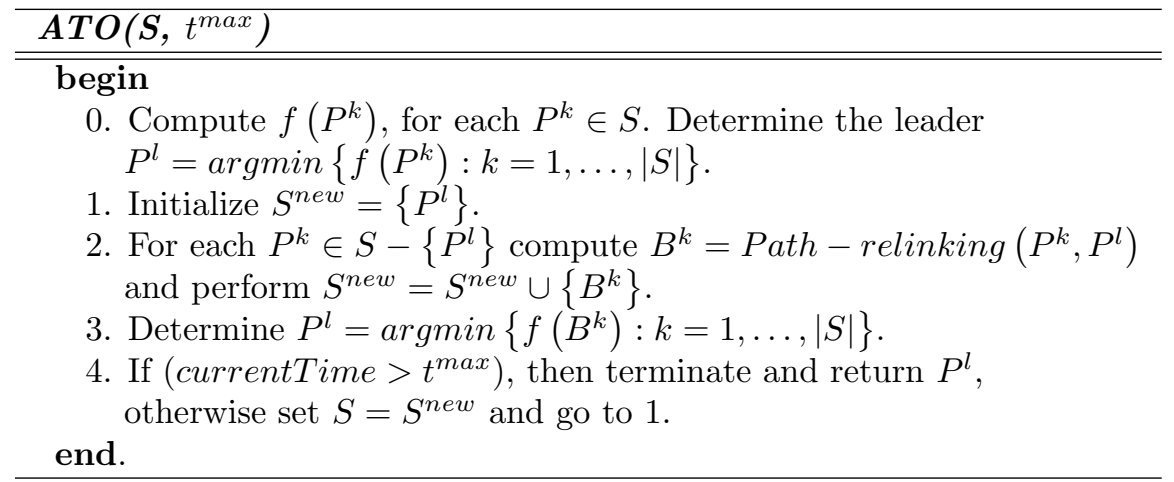

Table 2: $\operatorname{ATO}\left(S, t^{\max }\right)$

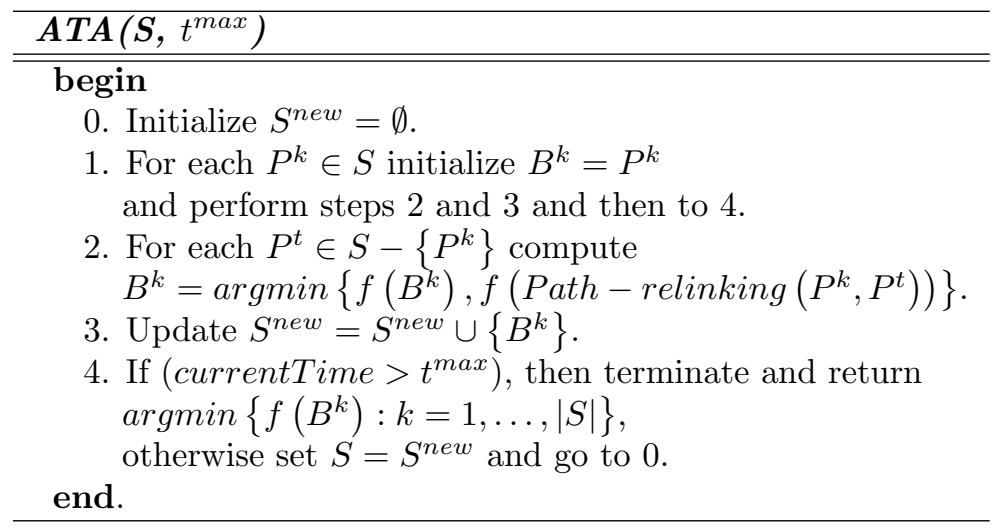

Table 3: $\operatorname{ATA}\left(S, t^{\max }\right)$

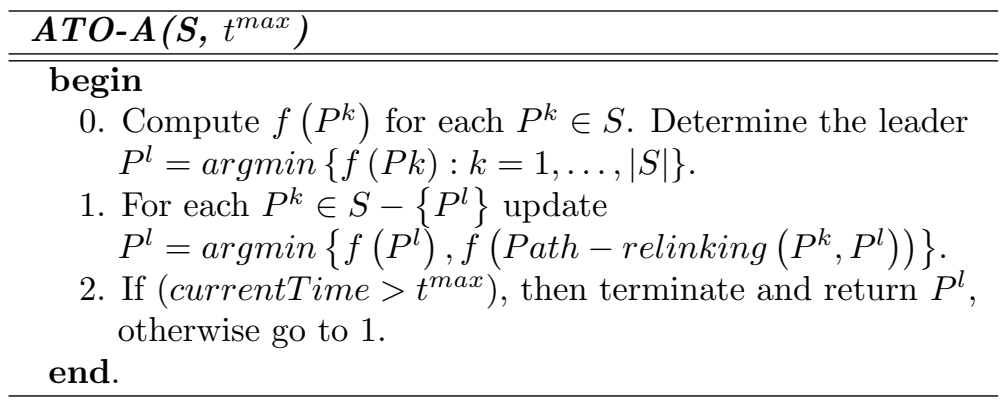

Table 4: $A T O-A\left(S, t^{\max }\right)$ 


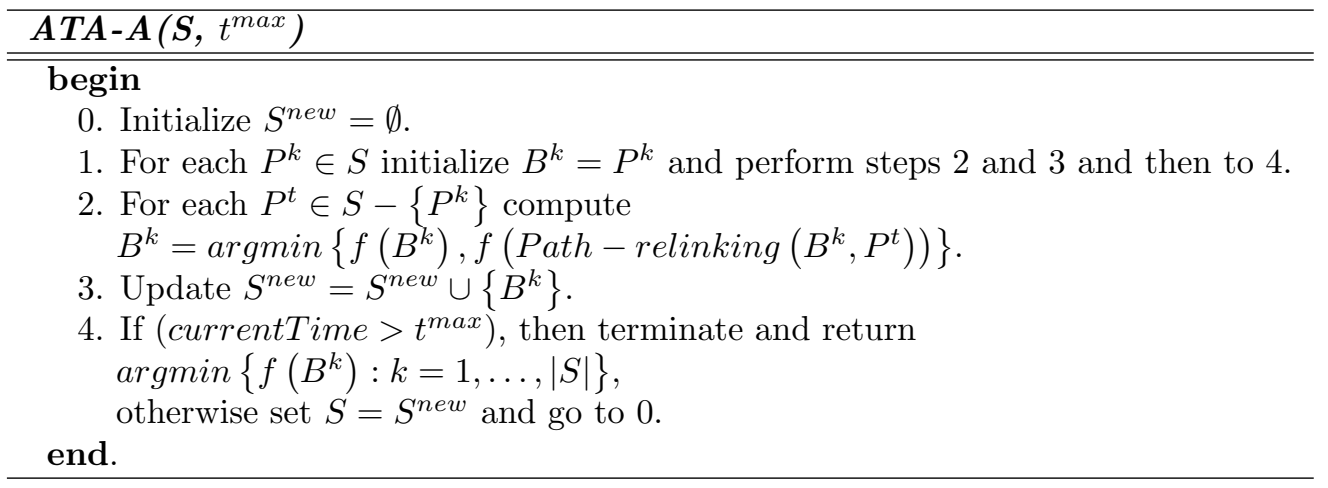

Table 5: $A T A-A\left(S, t^{\max }\right)$

\section{Numerical experiments}

The main goal of the following computational study is a comparison of the presented strategies of the discrete self-organizing migrating algorithm (DSOMA) with the goal to find the most efficient implementation of the algorithm for solving the generalized weighted $p$-median problem. The initial study has been performed using the benchmarks, which were derived from some of the Slovak self-governing regions. The individual instances of the benchmarks are denoted by the following names: Nitra (NR), Trenčín (TN), Trnava (TT), Žilina (ZA), Banská Bystrica (BB), Košice (KE) and Prešov (PO). The sizes of the individual benchmarks are determined by integers $m$ and $p$. The number $m$ gives the number of possible center locations and $p$ gives the number of service centers to be located. The generalized disutility objective function was computed for $r=3$. The coefficients $q_{k}$ for $k=1, \ldots, r$ have been obtained from statistics presented in [17] and their values are: $q_{1}=77.063, q_{2}=16.476$ and $q_{3}=100-q_{1}-q_{2}$.

The starting population for each solved instance was established as a uniformly deployed set of $p$-location problem solutions according to [14] and [15]. To increase robustness of the comparison, we made use of the uniformly deployed set property that each permutation of $m$ subscripts of locations generates a different uniformly deployed set. Thus, we generated randomly ten different uniformly deployed sets for each benchmark.

The sizes $m$ and $p$ and uniformly deployed set parameters $|S|$ and $h$ are reported in Table 1 together with the optimal solution values of (1) taken from [14]. The optimal solution values are reported in the column denoted by $O p t S o l$. The symbol $|S|$ denotes the cardinality of the set $S$ and $h$ is the minimal Hamming distance between each two problem solutions of the set.

\begin{tabular}{|c|cc|cc|c|}
\hline Region & $m$ & $p$ & $|S|$ & $h$ & OptSol \\
\hline \hline NR & 350 & 27 & 83 & 50 & 48940 \\
TN & 276 & 21 & 137 & 38 & 35275 \\
TT & 249 & 18 & 212 & 32 & 41338 \\
ZA & 315 & 29 & 112 & 52 & 42110 \\
BB & 515 & 36 & 172 & 66 & 44752 \\
KE & 460 & 32 & 60 & 60 & 45588 \\
PO & 664 & 32 & 232 & 60 & 56704 \\
\hline
\end{tabular}

Table 6: Benchmark parameters

Experiments with the individual strategies were organized so that each implementation was applied to each uniformly deployed set for given value of $t^{\max }$ and then the results were summarized in the table associated with the time threshold $t^{\max }$. A table for given $t^{\max }$ 


\begin{tabular}{|c|c|c|c|c|c|c|c|c|}
\hline \multirow[b]{3}{*}{ Region } & \multicolumn{4}{|c|}{$t^{\max }=10 \mathrm{~s}}$. & \multicolumn{4}{|c|}{$t^{\max }=5 s$} \\
\hline & \multicolumn{2}{|c|}{$\overline{A T O}$} & \multicolumn{2}{|c|}{$A T O-A$} & \multicolumn{2}{|c|}{$A T O$} & \multicolumn{2}{|c|}{$A T O-A$} \\
\hline & $F^{* a v g}$ & $C T[\mathrm{~s}]$ & $F^{* a v g}$ & $C T[\mathrm{~s}]$ & $F^{* a v g}$ & $C T[\mathrm{~s}]$ & $F^{* a v g}$ & $C T[\mathrm{~s}]$ \\
\hline NR & 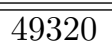 & $\bar{~} 10.0$ & 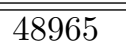 & $\overline{10.7}$ & 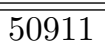 & $\overline{5.1}$ & 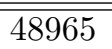 & $\overline{\overline{7.4}}$ \\
\hline $\mathrm{TN}$ & 35533 & 10.0 & 35277 & 11.5 & 35533 & 5.0 & 35277 & 7.0 \\
\hline TT & 41521 & 10.0 & 41386 & 10.8 & 41521 & 5.0 & 41386 & 6.6 \\
\hline $\mathrm{ZA}$ & 42314 & 10.0 & 42121 & 14.4 & 49807 & 5.2 & 42121 & 5.2 \\
\hline $\mathrm{BB}$ & 56510 & 25.0 & 44752 & 25.3 & 56510 & 26.1 & 44752 & 26.1 \\
\hline $\mathrm{KE}$ & 46070 & 10.0 & 45661 & 10.9 & 55502 & 5.9 & 45678 & 6.0 \\
\hline $\mathrm{PO}$ & 72673 & 33.4 & 56736 & 33.3 & 72673 & 35.2 & 56736 & 34.9 \\
\hline
\end{tabular}

Table 8: Results of numerical experiments with all-to-one strategies

contains sections denoted by $\boldsymbol{A T O}, \boldsymbol{A T A}, \boldsymbol{A T O}-\boldsymbol{A}, \boldsymbol{A T A}-\boldsymbol{A}$, in which the results obtained by the associated strategies are plotted. Each section contains two columns denoted by $F^{* a v g}$ and $C T[\mathrm{~s}]$. The column denoted by $F^{* a v g}$ reports the average minimum of the objective function of resulting solutions obtained by the approach application to ten uniformly deployed sets. The average computational times of the studied approaches are reported in the columns denoted by $C T[\mathrm{~s}]$. These values are given in seconds. As concerns the threshold $t^{\max }$ and reported computational time $C T$, we have to notice that the termination rule acts only after the new population is completed. Taking into account the computational complexity of the population update under the individual strategies, the threshold $t^{\max }$ can be several times exceeded in all-to-all strategies. That is why computational time is reported regardless of the threshold setting. The experiments were performed for $t^{\max }=10$ seconds. The numerical experiments were run on a PC equipped with the Intel@ Core $^{T M}$ i7 3610QM processor with the parameters: $2.3 \mathrm{GHz}$ and $8 \mathrm{~GB}$ RAM. The algorithms were implemented in the Java language and run in the NetBeans IDE 8.2 environment.

\begin{tabular}{|c|cc|cc|cc|cc|}
\hline & \multicolumn{2}{|c|}{$\boldsymbol{A T \boldsymbol { O }}$} & \multicolumn{2}{c|}{$\boldsymbol{A T A}$} & \multicolumn{2}{c|}{$\boldsymbol{A T \boldsymbol { A } \boldsymbol { A }}$} & \multicolumn{2}{c|}{$\boldsymbol{A T \boldsymbol { A } - \boldsymbol { A }}$} \\
Region & $F^{* a v g}$ & CT $[\mathrm{s}]$ & $F^{* \text { avg }}$ & $C T[\mathrm{~s}]$ & $F^{* a v g}$ & $C T[\mathrm{~s}]$ & $F^{* a v g}$ & $C T[\mathrm{~s}]$ \\
\hline \hline $\mathrm{NR}$ & 49320 & 10.0 & 57556 & 300.1 & 48965 & 10.7 & 48961 & 149.8 \\
$\mathrm{TN}$ & 35533 & 10.0 & 42473 & 316.3 & 35277 & 11.5 & 35276 & 159.4 \\
$\mathrm{TT}$ & 41521 & 10.0 & 46528 & 456.0 & 41386 & 10.8 & 41386 & 230.6 \\
ZA & 42314 & 10.0 & 49025 & 562.3 & 42121 & 14.4 & 42120 & 283.6 \\
\hline
\end{tabular}

Table 7: Results of numerical experiments for the threshold $t^{\max }=10$ seconds

Having compared the preliminary results reported in Table 7, we have found that the both all-to-all strategies are very inefficient concerning the type of studied problem and the way of creating the starting population. In the prescribed time $t^{\max }=10$ seconds, these strategies were not able to complete a single population update. The update takes an order of magnitude higher time than the threshold. Despite this extension, the results of AT A differ significantly from the optimal solution, while the other strategies reach almost the optimal solutions with accuracy better than one percent of the optimal value. It can be noticed that the adaptive mode considerably contributes to the resulting solution quality. As the all-to-all approach represents such a burden in a strategy performance that it demands for inacceptable computational time, we restricted the remainder of the computational study only to all-to-one strategies. We applied them to a broader set of benchmarks and, furthermore, we reduced the time threshold to estimate differences in their convergence speed. The obtained results are plotted in Table 8. 
The results reported in Table 8 confirm advantage of the adaptive mode over the simple all-to one strategy. Even if the ATO strategy is able to repeat the population update more times in the given threshold compared to $A T O-A$, the $A T O-A$ results are better even for the harder instances BB and PO.

\section{Conclusion}

The paper deals with possible strategies of the self-organizing migrating algorithm and follows the goal to find the most suitable strategy for the generalized weighted $p$-median problem using a uniformly deployed set of solutions as the starting population. We have performed a series of numerical experiments and found that the most suitable strategy for the given type of problem and the special way of starting population determination is the adaptive all-to one strategy. We confirmed the hypothesis that it is possible to suggest a sophisticated heuristic for a specific problem, which can produce near-to-optimal solution in much smaller time than the time demanded by an exact method.

Further research in this area may be focused on exploitation the preliminary mapping of the feasible solution set in the range of objective function values yielded by the uniformly deployed set for accelerating the self-organizing migrating algorithm performance. The next objective of the future research will be aimed at applications of the suggested method to obtaining series of non-dominated solutions of the bi-criterial $p$-location problems.

\section{Acknowledgements}

This work was supported by the research grants VEGA1/0089/19 "Data analysis methods and decisions support tools for service systems supporting electric vehicles", and VEGA 1/0689/19 "Optimal design and economically efficient charging infrastructure deployment for electric buses in public transportation of smart cities". This work was supported by the Slovak Research and Development Agency under the Contract no. APVV-19-0441.

\section{References}

[1] Avella, P., Sassano, A., and Vasil'ev, I. (2007). Computational study of large scale p-median problems. Mathematical Programming, 109(1), 89-114. doi: 10.1007/s10107-005-0700-6

[2] Clerc, M. (2006). Particle Swarm Optimization. ISTE Ltd., p. 243.

[3] Current, J., Daskin, M., and Schilling, D. (2002). Discrete network location models. Drezner Z. et al. (Eds.) Facility location: Applications and theory (pp. 81-118). Springer International Publishing.

[4] Čičková, Z., Brezina, I., Pekár, J. (2016). Solving the Routing Problems with Time Windows. Davendra D., Zelinka, I. (Eds.) Self-Organizing Migrating Algorithm-Methodology and Implementation (pp. 207-236). Springer International Publishing.

[5] Davendra, D. and Zelinka, I. (2016). Self-Organizing Migrating Algorithm, Methodology and Implementation. Springer International Publishing.

[6] Deep K. and Singh D. (2016). Optimization of Directional Overcurrent Relay Times. Davendra D., Zelinka, I. (Eds.) Self-Organizing Migrating Algorithm-Methodology and Implementation (pp. 167-186). Springer International Publishing.

[7] Doerner, K. F., Gutjahr, W. J., Hartl, R. F., Karall, M. and Reimann, M. (2005). Heuristic Solution of an Extended Double-Coverage Ambulance Location Problem for Austria. Central European Journal of Operations Research, 13(4), 325-340.

[8] Drezner, T. and Drezner, Z. (2007). The gravity p-median model. European Journal of Operational Research, 179, 1239-1251. doi:10.1016/j.ejor.2005.04.054

[9] Elloumi, S., Labbé, M. and Pochet, Y. (2004). A new formulation and resolution method for the p-center problem. INFORMS Journal on Computing, 16(1), 84-94. doi:10.1287/ijoc.1030.0028 
[10] García, S., Labbé, M. and Marín, A. (2011). Solving large p-median problems with a radius formulation. INFORMS Journal on Computing, 23(4), 546-556. doi:10.1287/ijoc.1100.0418

[11] Gendreau, M. and Potvin, J. (2010). Handbook of Metaheuristics. Springer Science \& Business Media. doi:10.1007/978-1-4419-1665-5

[12] Grygar D. and Fabricius R. (2019). An Efficient Adjustment of Genetic Algorithm for Pareto Front Determination. Transportation Research Procedia, 40, 1335-1342. doi:10.1016/j.trpro.2019.07.185

[13] Janáček, J. and Kvet, M. (2016). Min-max Optimization and the Radial Approach to the Public Service System Design with Generalized Utility. Croatian Operational Research Review, 7(1), 4961. doi:10.17535/crorr.2016.0005

[14] Janáček, J. and Kvet, M. (2019). Uniform Deployment of the p-Location Problem Solutions. Operations Research Proceedings 2019, Springer International Publishing, in print.

[15] Janáček, J. and Kvet, M. (2019). Usage of Uniformly Deployed Set for p-Location Min-Sum Problem with Generalized Disutility. SOR 2019 Proceedings, 494-499.

[16] Janáček, J. and Kvet, M. (2020). Adaptive Path-Relinking Method for Public Service System Design. In: Mathematical Methods in Economics 2020, in print.

[17] Jankovič, P. (2016). Calculating Reduction Coefficients for Optimization of Emergency Service System Using Microscopic Simulation Model. 17th International Symposium on Computational Intelligence and Informatics, 163-167.

[18] Jánošíková, Ľ. and Žarnay, M. (2014). Location of emergency stations as the capacitated p-median problem. Quantitative Methods in Economics (Multiple Criteria Decision Making XVII), 117-123.

[19] Kvet, M. (2014). Computational Study of Radial Approach to Public Service System Design with Generalized Utility. Proceedings of International Conference Digital Technologies 2014, 198-208.

[20] Kvet, M., Janáček, J. (2020). Path-Relinking Method for Weighted p-Median Problem Solutions. In: Quantitative Methods in Economics 2020, in print.

[21] Marianov, V. and Serra, D. (2002). Location problems in the public sector, Drezner Z. et al. (Eds.) Facility location: Applications and theory (pp. 119-150), Springer International Publishing.

[22] Pekár, J., Čičková, Z., Brezina, I. (2016). Some in Financial Modeling, Davendra D., Zelinka, I. (Eds.) Self-Organizing Migrating Algorithm-Methodology and Implementation (pp. 237-253), Springer International Publishing.

[23] Rybičková, A., Burketová, A. and Mocková, D. (2016). Solution to the locating - routing problem using a genetic algorithm. SmaRTT Cities Symposiuum Prague, 1-6.

[24] Sayah, D. and Irnich, S. (2016). A new compact formulation for the discrete p-dispersion problem. European Journal of Operational Research, 256(1), 62-67. doi:10.1016/j.ejor.2016.06.036

[25] Singh D. and Deep K. (2016). SOMGA for Large Scale Function Optimization, Davendra D., Zelinka, I. (Eds.) Self-Organizing Migrating Algorithm-Methodology and Implementation (pp. 187-206), Springer International Publishing.

[26] Snyder, L. V. and Daskin, M. S. (2005). Reliability models for facility location; The expected failure cost case. Transport Science, 39(3), 400-416. doi:10.1287/trsc.1040.0107

[27] Yilmaz, O. (2020). Attaining flexibility in seru production system by means of Shojinka: An optimization model and solution approaches. Computers \& Operations Research, 119, 104917. doi:10.1016/j.cor.2020.104917

[28] Yilmaz, O. and Durmusoglu, M. (2019). Multi-objective scheduling problem for hybrid manufacturing systems with walking workers. The International Journal of Industrial Engineering: Theory, Applications and Practice, 26, 625-650. journals.sfu.ca/ijietap/index.php/ijie/article/view/2810

[29] Yilmaz, O., Ozcelik, G., Yeni, F.B. (2019). Lean holistic fuzzy methodology employing cross-functional worker teams for new product development projects: A real case study from high-tech industry. European Journal of Operational Research, 282(3), 989-1010. doi:10.1016/j.ejor.2019.09.048

[30] Zelinka, I. (2016). SOMA-Self -organizing Migrating Algorithm, Davendra D., Zelinka, I. (Eds.) Self-Organizing Migrating Algorithm-Methodology and Implementation (pp. 3-49), Springer International Publishing. 